Article

\title{
Temperature Stability of the Sky Quality Meter
}

\author{
Sabrina Schnitt ${ }^{1}$, Thomas Ruhtz ${ }^{1}$, Jürgen Fischer ${ }^{1}$, Franz Hölker ${ }^{2}$ \\ and Christopher C.M. Kyba ${ }^{1,2, *}$
}

${ }^{1}$ Institute for Space Sciences, Freie Universität Berlin, Carl-Heinrich-Becker-Weg 6-10, Berlin 12165, Germany; E-Mails: Sabrina.schnitt@fu-berlin.de (S.S.); ruhtz@ zedat.fu-berlin.de (T.R.); juergen.fischer@wew.fu-berlin.de (J.F.)

${ }^{2}$ Leibniz-Institute of Freshwater Ecology and Inland Fisheries, Müggelseedamm 310, Berlin 12587, Germany; E-Mail: hoelker@igb-berlin.de

* Author to whom correspondence should be addressed; E-Mail: christopher.kyba@ wew.fu-berlin.de; Tel.: +49-30-838-71140.

Received: 23 August 2013; in revised form: 6 September 2013 / Accepted: 9 September 2013 / Published: 11 September 2013

\begin{abstract}
The stability of radiance measurements taken by the Sky Quality Meter (SQM) was tested under rapidly changing temperature conditions during exposure to a stable light field in the laboratory. The reported radiance was found to be negatively correlated with temperature, but remained within $7 \%$ of the initial reported radiance over a temperature range of $-15^{\circ} \mathrm{C}$ to $35^{\circ} \mathrm{C}$, and during temperature changes of $-33^{\circ} \mathrm{C} / \mathrm{h}$ and $+70{ }^{\circ} \mathrm{C} / \mathrm{h}$. This is smaller than the manufacturer's quoted unit-to-unit systematic uncertainty of $10 \%$, indicating that the temperature compensation of the SQM is adequate under expected outdoor operating conditions.
\end{abstract}

Keywords: light pollution; sky quality meter; light at night; temperature; stability; skyglow; night sky brightness

\section{Introduction}

Skyglow is the diffuse light of the sky over cities at night that occurs because artificial light emitted upward from the city scatters back towards the ground. Until very recently, skyglow had only been measured at a handful of locations around the world (e.g., [1,2]). In the last few years, however, studies 
of skyglow have been undertaken over large areas [3-6], and time series have been produced under different meteorological and celestial conditions [7-15].

Two of the reasons for this increased monitoring are the recognition of the biological importance of darkness, and continued growth in the amount of lighting worldwide [16]. Exposure to unnatural levels of light at night can have ecosystem level effects [17], and can alter physiological processes and hormone levels in many organisms (including humans), presumably leading to undesirable health outcomes [18]. While the strongest biological effects of artificial lighting take place directly under the lamps, the diffuse skyglow over cities may also be important [17-19]. For example, skyglow can change the behavior [20] of organisms, and can impair the navigational ability of organisms that depend upon celestial signals [19,21-23].

An additional reason for the increased monitoring is that quantitative studies of the night sky became easier to perform with the development of low-cost light meters: The "International Year of Astronomy Lightmeter" (IYAL), [10] produced by K2WLights, and the "Sky Quality Meter" (SQM), manufactured by Unihedron. Most of the studies referenced above made use of the SQM.

The response of the SQM to light was characterized by Cinzano [24] for a handheld SQM in laboratory conditions. In order to make scientific conclusions based on different skyglow meters at different locations, it is necessary to know the systematic uncertainty of the values reported by the devices. Different groups have set up experiments to track the meter-to-meter differences [25] and long term stability [11] of SQMs, but the temperature stability of the SQM has not yet been investigated.

The SQM is almost always installed and used out-of-doors, and therefore experiences fairly large temperature fluctuations. Since both ambient temperature and temperature changes can affect the response of light collecting elements and the properties of electronic components, it is prudent to check whether and to what extent SQMs are affected by temperature, and by fluctuations in temperature. In this paper, we investigate the response of two SQMs exposed to a constant light field under conditions of changing temperature in the laboratory.

\section{Methods}

\subsection{Sky Quality Meter}

The SQM measures sky radiance in the logarithmic units of $\operatorname{mag}_{\mathrm{SQM}} / \mathrm{arcsec}^{2}$, which are often used by astronomers (the subscript SQM indicates that the spectral sensitivity of the SQM is not identical to the $\mathrm{V}$ band). The field of view (FOV) is $\sim 20^{\circ}$ (full width at half maximum) for the SQM-LU (Lens and USB readout) devices considered in this paper. To aid interpretation, all measurements have been converted to values relative to the radiance observed at the start of the measurements, using the formula $10^{0.4 \Delta_{M}}$, where $\Delta_{M}$ is the difference between the current and starting measurement in $\mathrm{mag}_{\mathrm{SQM}} / \mathrm{arcsec}^{2}$. The starting radiance in each measurement was approximately $19 \mathrm{mag}_{\mathrm{SQM}} / \mathrm{arcsec}^{2}$, which is a typical value for the cloud-free night sky above our laboratory in Steglitz, Berlin, Germany [8]. 
The SQM is not weatherproof, and its response can be affected if water enters the device (personal communication from Unihedron). It must therefore be protected from moisture by installing it in a housing, and in our experience, most users have chosen to use the housing provided by the manufacturer shown in Figure 1. The SQM housing not only protects the device from the environment but also traps the heat generated by the operation of the device. This heating is negligible in the case of SQMs connected via USB (SQM-LU), but significant in the case of Ethernet connected SQMs (SQM-LE), which tend to operate at slightly warmer temperatures. To avoid the possibility of Ethernet heating interfering with the tests, in this paper only the USB version of the SQM (SQM-LU) is studied.

Figure 1. The Sky Quality Meter and the standard housing. At right is an Ethernet based SQM-LE (two cables) that is not installed inside of its housing. When the SQM is installed in the housing it appears as at left (a USB based SQM-LU with only one cable). The housing protects the SQM from the elements, and in the case the SQM-LE slightly raises the temperature relative to the surroundings. Image available under the CC BY 2.5 license. doi:10.1371/journal.pone.0017307.g002.

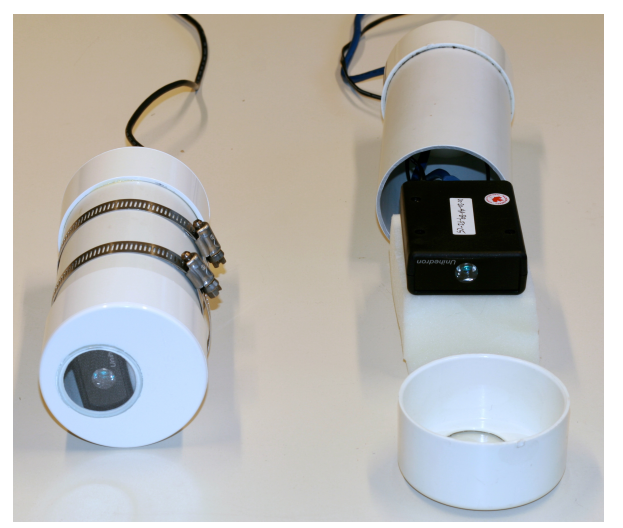

The response of the light sensor of the SQM (TAOS TSL237S) has a well characterized bias with temperature, which is reported by the manufacturer. The SQM's internal software attempts to account for this bias by monitoring the temperature near the light sensor, and correcting the observed signal according to the expected bias. The temperatures that can be expected to be encountered by an SQM in the field will depend on the measurement location and time of year. In the period from 1 April 2010 to 19 August 2013 in Berlin, Steglitz, an SQM-LU mounted on the roof of our institute observed a temperature range from $-16.5^{\circ} \mathrm{C}$ to $30.3{ }^{\circ} \mathrm{C}$ during darkness. The median rate of temperature change was $-0.3{ }^{\circ} \mathrm{C} / \mathrm{h}$, and the most extreme rates of temperature change observed were $-9.0^{\circ} \mathrm{C} / \mathrm{h}$ and $+2.6^{\circ} \mathrm{C} / \mathrm{h}$.

\subsection{Testing Temperature Stability}

The temperature stability of the SQMs was tested by installing them inside of a programmable temperature chamber (Weiss Umwelttechnik, 125SB) designed for materials testing. Two devices were tested simultaneously, one free and one in the standard Unihedron housing as shown in Figure 2. One porthole allowed the passage of cables into the chamber, another one was left open and exposed to light from a stable, diffuse source (integrating sphere by Lichtmesstechnik Berlin). To reduce the radiance to typical values for an urban night sky, the light from the integrating sphere was incident on a dark curtain 
hung inside of a temperature chamber. The SQMs were manually arranged to ensure a starting radiance between 18.9 and $19.1 \mathrm{mag}_{\mathrm{SQM}} / \operatorname{arcsec}^{2}$. The stability of the light source was verified by monitoring the SQMs over a period of several days with the power to the chamber off (i.e., room temperature). No variation in measured radiance was observed in either of the two SQMs.

Figure 2. The temperature chamber used for testing the light meters is shown, with two SQM-LU devices in place. The white cylinder is the standard SQM external housing, and fixed to it is a free SQM-LU. The view direction of the SQMs is towards the right, where a dark curtain reduces the radiance to typical night sky values above our laboratory. A USB temperature logger is located behind the free SQM-LU. Light enters from a porthole on the left.

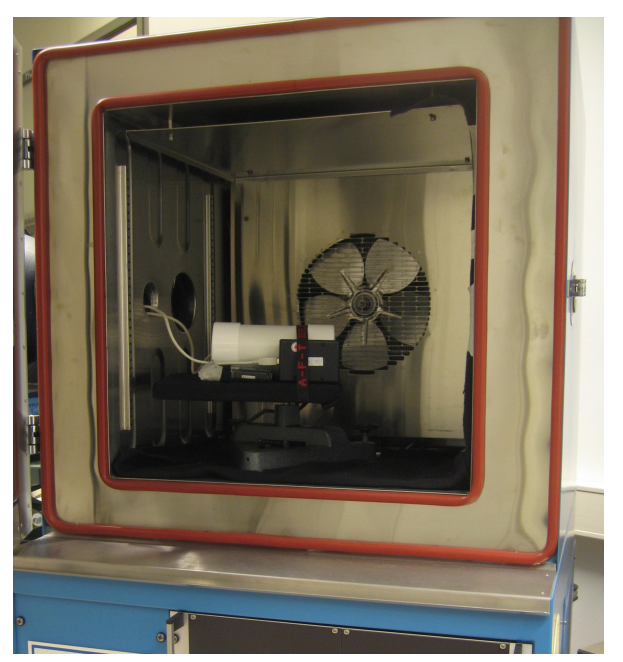

In order to test the response of the device at different stable temperatures, the temperature chamber was programmed to run a series of temperature ramps over two days, with long pauses between the ramps (Test 1). The temperature end points and rates of change of the ramps are shown in Table 1. The device's response to extremely rapid temperature change was tested with a "stress program", which was repeated three times (Test 2). The rates of temperature change and end points of Test 2 are shown in Table 2. The chamber's temperature was monitored using the thermometers built into the SQM-LUs, as well as a USB temperature logger (Voltcraft DL-120TH). The SQMs were read out every second, and the reported radiances were averaged over $60 \mathrm{~s}$ intervals.

Table 1. Temperature and temperature change parameters for Test 1.

\begin{tabular}{cccccccccc}
\hline Time $(\mathbf{h})$ & $0-12$ & $12-15$ & $15-18$ & $18-25$ & $25-30$ & $30-34$ & $34-39$ & $39-40$ & $40-43$ \\
\hline Temperature $\left({ }^{\circ} \mathrm{C}\right)$ & 20 & & 35 & & -15 & & 35 & & 20 \\
Temp change $\left({ }^{\circ} \mathrm{C} / \mathrm{h}\right)$ & 0 & +5 & 0 & -7.1 & 0 & +12.5 & 0 & -15 & 0 \\
\hline
\end{tabular}


Table 2. Temperature and temperature change parameters for Test 2.

\begin{tabular}{ccccc}
\hline Time $(\mathbf{h})$ & $0-1$ & $1-2.5$ & $2.5-4$ & $4-4.5$ \\
\hline Temperature aim $\left({ }^{\circ} \mathrm{C}\right)$ & 20 & 35 & -15 & 20 \\
Temp change $\left({ }^{\circ} \mathrm{C} / \mathrm{h}\right)$ & 0 & +10 & -33.3 & +70 \\
\hline
\end{tabular}

The temperature chamber was not designed for optical measurements, and vibrated during operation. Early testing indicated that the pointing direction of the SQMs could shift during operation. The light field on the dark curtains was not perfectly uniform, so a shift in optical alignment would result in a change in observed radiance. For this reason, clamps and a heavy support plate were used to minimize this motion. The humidity of the chamber was not controlled, and dew was occasionally noted on the devices after the rapid temperature shift program completed. This could potentially have some effect on the optics, and therefore affect the observed radiance.

\section{Results and Discussion}

The results of the temperature stability test (Test 1) for the two SQM-LU devices are shown in Figure 3. The upper plot shows the change in radiance relative to the initial observation over the course of the two day test for the free SQM (red) and the SQM in the housing (green). The lower plot shows the chamber temperature as measured by the SQMs and the USB temperature monitor (blue). Both SQMs had qualitatively the same deviation behavior due to rapid temperature changes. The devices reported decreased radiance at higher temperatures, and an increased radiance at lower temperatures. Once the temperature was stabilized, the reported radiance was also more stable. At the end of the test, the reported radiance for both SQMs was about $2 \%$ lower than at the start, which very likely indicates a change in optical alignment. We performed repeated measurements with the SQM inside and outside of the housing swapped, and found that the qualitative response of the SQM inside the housing was relatively consistent. The response of the free SQM was less consistent, but in no case did the reported radiance change by more than $7 \%$ relative to the starting radiance.

The data for the rapid temperature change experiment (Test 2) are shown in Figure 4. Qualitatively, the results are very similar to Test 1 , and the response in each of the three cycles is very similar. The deviation in reported radiance ranged from $-4 \%$ to $+7 \%$ over the course of the test. At the end of the test, the devices differed by plus and minus $1 \%$ respectively, again indicating a slight change in the positioning of the SQMs. 
Figure 3. The observed deviation from the original radiance during the long temperature stability test (Test 1) is shown at top for a free SQM-LU (red), and for an SQM-LU inside of the standard weatherproof housing (green). The lower plot shows the temperature curve measured by the SQMs and the USB thermometer (blue). Dashed vertical lines show the time when temperature ramps were started or stopped.

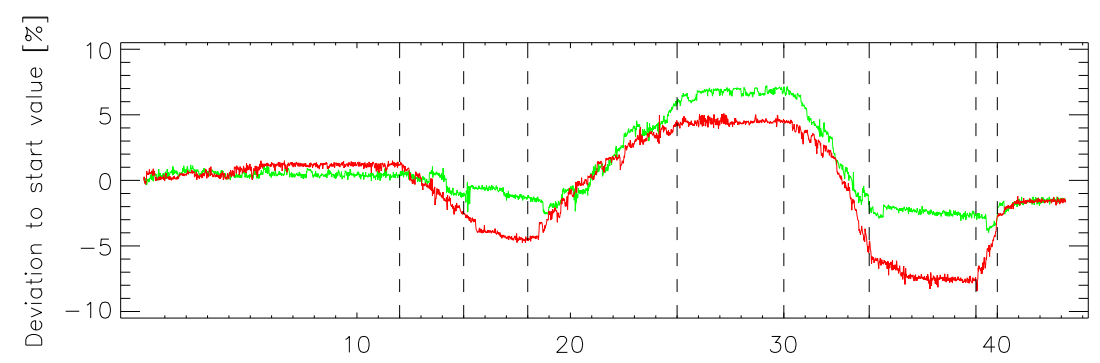

-- : USB thermometer
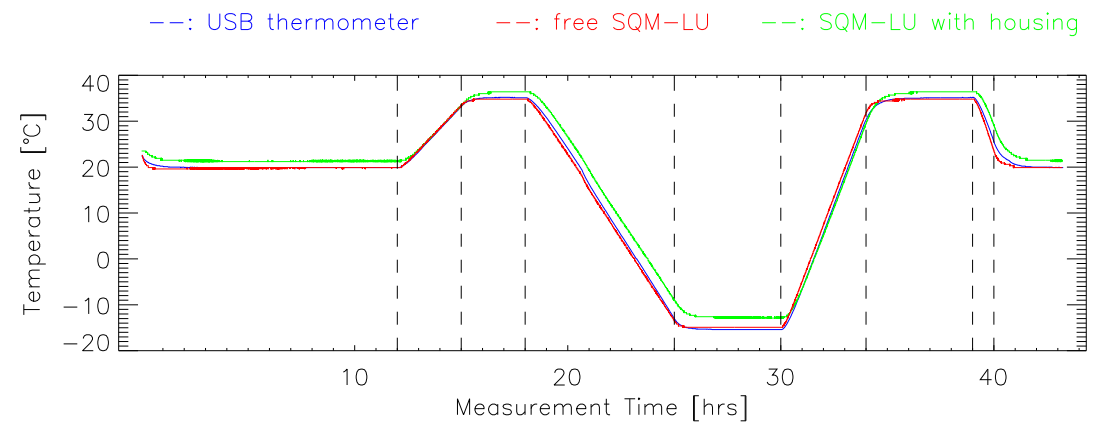

Figure 4. The observed deviation from the original radiance is shown for the shorter temperature stress program, which was repeated three times. The free SQM-LU is shown in red, the SQM-LU inside of the standard weatherproof housing in green. The lower graph shows the temperature curve measured by the SQMs and the USB thermometer (blue).

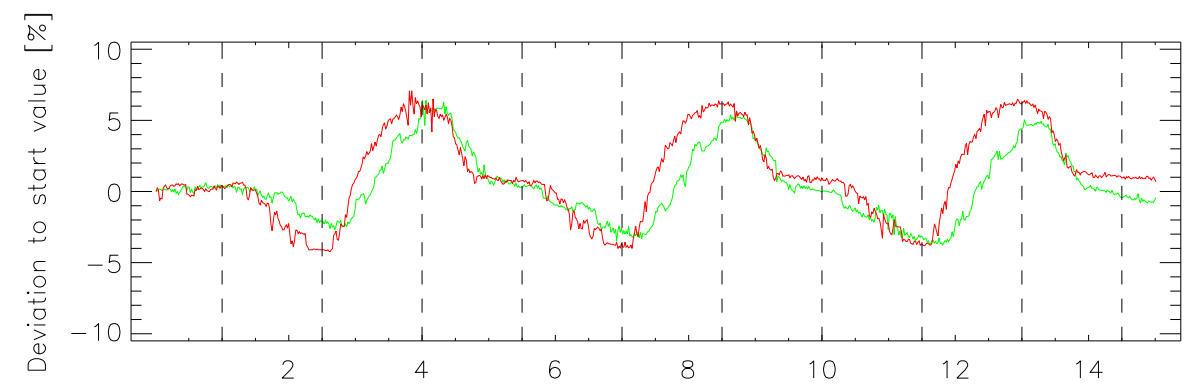

--: USB thermometer $\quad--$ : free SQM-LU $\quad--$ : SQM - LU with housing

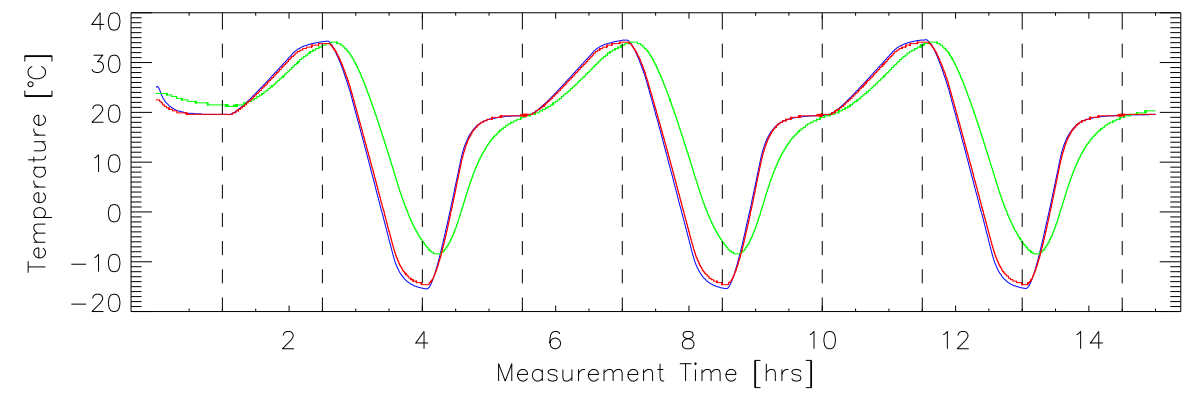


Due to the optical limitations of the experimental setup, it is unclear whether the observed changes in radiance are due to changes in the view direction or incorrect temperature compensation of the device. Under the assumption that most of the observed change is due to a failure in temperature compensation, it is possible to derive a correction factor. The average deviation in one of the SQMs was reasonably well matched to a linear fit, while the second SQM was better described with a second degree polynomial. For the sake of simplicity, an average linear correction for all four datasets was calculated. Reported radiance could be approximately corrected using the formula:

$$
\mathrm{SQM}_{\text {corr }}=\mathrm{SQM}_{\text {rep }}-0.042+2.12 \times 10^{-3} \times T
$$

where $\mathrm{SQM}_{\text {rep }}$ and $\mathrm{SQM}_{\text {corr }}$ are the reported and corrected radiance in $\mathrm{mag}_{\mathrm{SQM}} / \operatorname{arcsec}^{2}$, and $T$ is the temperature reported by the SQM. Applying this correction factor to the data presented in Figures 3 and 4 reduced the worst case deviation (red curve in Figure 3) from $-7 \%$ to $+4 \%$ for $\mathrm{SQM}_{\text {rep }}$ to $-5 \%$ to $+1 \%$ for $\mathrm{SQM}_{\text {corr }}$.

With a sample of only two SQMs, it is not possible to conclude whether the correction function defined above applies equally well to all SQM devices. In addition, given that it is known that the alignment of the SQMs changed slightly during running, the correction equation provided above should be used only as an approximate guide in estimating uncertainties for measurements taken at different times with an identical meter. The uncorrected deviation reported here is small in comparison with the manufacturer's much larger quoted unit-to-unit systematic uncertainty in reported radiance of $\pm 10 \%$ (which we presume represents a standard deviation, and not a maximum range of differences). It is conceivable that an improved temperature compensation algorithm based on a larger scale study by the SQM manufacturer could reduce some of the measurement uncertainty.

The results of this study should be expected to apply to both the SQM-LE, as well as hand-held SQMs, which have a similar internal design. A number of groups have used an automobile in order to perform hand-held SQM measurements at widely separated distances (e.g., [4]). For the case of a heated car during the winter, very rapid temperature changes could be expected. Therefore, it is advisable that in such a measurement campaign care is taken to maintain the SQM at as close to outdoor temperature as possible while in transit between sites.

\section{Conclusions}

The stability of radiance measurements taken by two SQM-LU low light radiance meters was tested over the temperature range $-15^{\circ} \mathrm{C}$ to $35^{\circ} \mathrm{C}$, and the variation in reported radiance was found to be smaller than the $10 \%$ unit-to-unit systematic uncertainty quoted by the manufacturer. These same results can reasonably be expected to apply for SQM-LE devices as well as for hand-held SQMs. When operated in urban areas, SQMs routinely observe differences in sky radiance of an order of magnitude due to the presence or absence of clouds [8]. The temperature compensation of the SQM is therefore found to be adequate for use in the field over the range of $-15^{\circ} \mathrm{C}$ to $35^{\circ} \mathrm{C}$. 


\section{Acknowledgments}

This work was supported by the project Verlust der Nacht (funded by the Federal Ministry of Education and Research, Germany, BMBF-033L038A) and by focal area MILIEU (FU Berlin).

\section{Conflicts of Interest}

The authors declare no conflicts of interest.

\section{References}

1. Walker, M. The California site survey. Publ. Astron. Soc. Pac. 1970, 82, 672-698.

2. Hoag, A.A. City sky glow monitoring at Kitt Peak. Pub. Astron. Soc. Pac. 1973, 85, 503-507.

3. Barringer, D.; Walker, C.E.; Pompea, S.M.; Sparks, R.T. Astronomy Meets the Environmental Sciences: Using GLOBE at Night Data. In Proceedings of the Earth and Space Science: Making Connections in Education and Public Outreach, Boulder, CO, USA, 31 July-4 August 2010.

4. Biggs, J.D.; Fouch, T.; Bilki, F.; Zadnik, M.G. Measuring and mapping the night sky brightness of Perth, Western Australia. Mon. Not. R. Astron. Soc. 2012, 421, 1450-1464.

5. Pun, C.; So, C. Night-sky brightness monitoring in Hong Kong: A city-wide light pollution assessment. Environ. Monit. Assess. 2012, 184, 2537-2557.

6. Kyba, C.C.M.; Wagner, J.M.; Kuechly, H.U.; Walker, C.E.; Elvidge, C.D.; Falchi, F.; Ruhtz, T.; Fischer, J.; Hölker, F. Citizen science provides valuable data for monitoring global night sky luminance. Sci. Rep. 2013, doi:10.1038/srep01835.

7. Falchi, F. Campaign of sky brightness and extinction measurements using a portable CCD camera. Mon. Not. R. Astron. Soc. 2011, 412, 33-48.

8. Kyba, C.C.M.; Ruhtz, T.; Fischer, J.; Hölker, F. Cloud coverage acts as an amplifier for ecological light pollution in urban ecosystems. PLoS One 2011, 6, e17307.

9. Lolkema, D.; Haaima, M.; den Outer, P.; Spoelstra, H. Effects of Meteorological and Atmospheric Parameters on Night Sky Brightness; Technical Report RIVM \#680151002; Netherlands National Institute for Public Health and the Environment, Bilthoven, The Netherlands, 2011.

10. Müller, A.; Wuchterl, G.; Sarazin, M. Measuring the night sky brightness with the lightmeter. Revista Mexicana de Astronoma y Astrofsica 2011, 41, 46-49.

11. Kyba, C.C.M.; Ruhtz, T.; Fischer, J.; Hölker, F. Red is the new black: How the color of urban skyglow varies with cloud cover. Mon. Not. R. Astron. Soc. 2012, 425, 701-708.

12. Ściężor, T.; Kubala, M.; Kaszowski, W. Light pollution of the mountain areas in Poland. Arch. Environ. Prot. 2012, 38, 59-69.

13. Davies, T.W.; Bennie, J.; Inger, R.; Gaston, K.J. Artificial light alters natural regimes of night-time sky brightness. Sci. Rep. 2013, doi:10.1038/srep01722.

14. Puschnig, J.; Posch, T.; Uttenthaler, S. Night sky photometry and spectroscopy performed at the Vienna University Observatory. J. Quant. Spectrosc. Radiat. 2013, in press. 
15. Ściężor, T. A new astronomical method for determining the brightness of the night sky and its application to study long-term changes in the level of light pollution. Mon. Not. R. Astron. Soc. 2013, in press.

16. Hölker, F.; Moss, T.; Griefahn, B.; Kloas, W.; Voigt, C.; Henckel, A.; Hänel, A.; Kappeler, P.; Völker, S.; Schwope, A.; et al. The dark side of light-A transdisciplinary research agenda for light pollution policy. Ecol. Soc. 2010, 15, Available online: http://www.ecologyandsociety.org/vol15/iss4/art13/ (accessed on 11 September 2013).

17. Rich, C.; Longcore, T. Ecological Consequences of Artificial Night Lighting; Island Press: Washington, DC, USA, 2006.

18. Navara, K.J.; Nelson, R.J. The dark side of light at night: Physiological, epidemiological, and ecological consequences. J. Pineal. Res. 2007, 43, 215-224.

19. Kyba, C.C.M.; Hölker, F. Do artificially illuminated skies affect biodiversity in nocturnal landscapes? Landsc. Ecol. 2013, in press.

20. Moore, M.V.; Pierce, S.M.; Walsh, H.M.; Kvalvik, S.K.; Lim, J.D. Urban light pollution alters the diel vertical migration of Daphnia. Verh. Internat. Verein. Limnol. 2000, 27, 779-782.

21. Dacke, M.; Nilsson, D.E.; Scholtz, C.H.; Byrne, M.; Warrant, E.J. Insect orientation to polarized moonlight. Nature 2003, 424, 33.

22. Kyba, C.C.M.; Ruhtz, T.; Fischer, J.; Hölker, F. Lunar skylight polarization signal polluted by urban lighting. J. Geophys. Res. 2011, 116, D24106.

23. Dacke, M.; Baird, E.; Byrne, M.; Scholtz, C.; Warrant, E. Dung beetles use the milky way for orientation. Curr. Biol. 2013, 23, 298-300.

24. Cinzano, P. Night Sky Photometry with Sky Quality Meter; Technical Report 9, ISTIL, 2005. v1.4.

25. Den Outer, P.; Lolkema, D.; Haaima, M.; van der Hoff, R.; Spoelstra, H.; Schmidt, W. Intercomparisons of nine sky brightness detectors. Sensors 2011, 11, 9603-9612.

(c) 2013 by the authors; licensee MDPI, Basel, Switzerland. This article is an open access article distributed under the terms and conditions of the Creative Commons Attribution license (http://creativecommons.org/licenses/by/3.0/). 